\title{
Chemokines in CSF of Alzheimer's disease patients
}

\author{
Jôice Dias Corrêa', Daniela Starling², Antônio Lúcio Teixeira², \\ Paulo Caramelli², Tarcília Aparecida Silva
}

\begin{abstract}
Some studies have linked the presence of chemokines to the early stages of Alzheimer's disease (AD). Then, the identification of these mediators may contribute to diagnosis. Our objective was to evaluate the levels of beta-amyloid (BA), tau, phospho-tau (p-tau) and chemokines (CCL2, CXCL8 and CXCL10) in the cerebrospinal fluid (CSF) of patients with $A D$ and healthy controls. The correlation of these markers with clinical parameters was also evaluated. The levels of $p$-tau were higher in AD compared to controls, while the tau/p-tau ratio was decreased. The expression of CCL2 was increased in AD. A positive correlation was observed between BA levels and all chemokines studied, and between CCL2 and p-tau levels. Our results suggest that levels of CCL2 in CSF are involved in the pathogenesis of $A D$ and it may be an additional useful biomarker for monitoring disease progression.
\end{abstract}

Key words: Alzheimer's disease, cerebrospinal fluid, chemokines, inflammation.

\section{Quimiocinas no LCR de pacientes portadores da doença de Alzheimer}

\section{RESUMO}

Alguns estudos têm relacionado a presença de quimiocinas com estágios iniciais da doença de Alzheimer (ALZ). A identificação desses mediadores pode contribuir para um diagnóstico precoce. O objetivo deste estudo foi avaliar os níveis de beta-amiloide (BA), tau, fosfo-tau (p-tau) e quimiocinas (CCL2, CXCL8 e CXCL10) no líquido cefalorraquidiano dos pacientes com ALZ e controles saudáveis e a correlação destes marcadores com parâmetros clínicos. Os níveis de p-tau foram maiores nos pacientes com ALZ em relação aos controles, enquanto a razão tau/p-tau foi menor. Houve um aumento significativo de CCL2. Uma correlação positiva foi encontrada entre os níveis de BA e todas as quimiocinas estudadas e também entre os níveis de CCL2 e p-tau. Nossos resultados sugerem que a presença de CCL2 está envolvida na patogênese da ALZ e que esta quimiocina pode ser um marcador adicional para monitorar a progressão da doença.

Palavras-chave: doença de Alzheimer, líquido cefalorraquidiano, quimiocinas, inflamação.

\section{Correspondence}

Tarcília Aparecida da Silva

Av. Antônio Carlos 6627

31270-901 Belo Horizonte MG - Brasil

E-mail: tarcilia@ufmg.br

\author{
Support \\ This work has been supported by \\ Fundação de Amparo a Pesquisa do \\ Estado de Minas Gerais (FAPEMIG, \\ Brazil, Grant 3247106) and Conselho \\ Nacional de Desenvolvimento Científico \\ e Tecnológico (CNPq, Brasil)
}

Received 30 November 2010 Received in final form 9 February 2011 Accepted 17 February 2011
The dementing diseases represent a growing medical, economic and social problem and Alzheimer's disease (AD) is the most common cause of dementia associated with advancing age ${ }^{1}$. AD is morphologically characterized by extracellular beta-amyloid (BA) plaque deposition, intraneuronal tau-pathology, neuronal cell death, vascular dysfunction and inflammatory processes ${ }^{1-3}$. Cerebrospinal fluid (CSF) closely reflects the composition of the brain extracellular space and is likely to have the highest yield of biomarkers for the evaluation of dementia ${ }^{4}$. Many studies reported elevated concentrations of tau proteins and decreased levels of BA peptides in the CSF of patients with $\mathrm{AD}^{5-7}$. Furthermore, it is widely accepted that a chronic inflammatory reaction plays an important role in the pathogenesis of $\mathrm{AD}^{8,9}$. The inflammatory components in $\mathrm{AD}$ include microglia and astrocytes, the 
complement system, and various inflammatory mediators including cytokines and chemokines ${ }^{2,10,11}$.

The chemokines constitute a large family of molecules that induces chemotaxis, leukocytes extravasation, and modulation of a variety of leukocytes functions ${ }^{12}$. There is growing evidence that chemokines and chemokine receptors are up-regulated in resident central nervous system (CNS) cells during $\mathrm{AD}$, which may contribute to plaque-associated inflammation and neurodegeneration ${ }^{13}$. Of these, CCL2 is a member of the CC chemokine family that plays a significant role in inflammatory processes, including atherosclerosis and neurodegenerative disorders ${ }^{14}$. CCL2 up regulation has been demonstrated in $\mathrm{AD}^{2}$.

CXCL8, a microglia-derived chemokine produced in response to pro-inflammatory signals such as BA, could be important for recruiting activated microglia into areas of the brain damaged during the AD pathological process ${ }^{12}$.

CXCL10 immunoreactivity was markedly increased in reactive astrocytes in $\mathrm{AD}$ brain. Astrocytes positive for CXCL10 were found to be associated with neuritic plaques and showed an apparently coordinated up-regulation of CCL4 ${ }^{4,15}$.

The definitive diagnosis of AD can be done only after the pathological examination of the brain; so, clinical diagnosis currently prevails. Auxiliary methods of diagnosis are valuable and can contribute to early diagnosis and improve the quality of life of these patients. Some studies have considered the presence of chemokines as a sign of early stages of $\mathrm{AD}^{4}$. Then, the identification of these mediators in CSF may contribute to early diagnosis and monitoring disease progression.

In the present study, we prospectively assessed the levels of BA, tau, phospho-tau (p-tau) and chemokines (CCL2, CXCL8 E CXCL10) in the CSF of patients with $\mathrm{AD}$ and healthy controls. Furthermore, the correlation of these markers with clinical parameters was assessed.

\section{METHOD}

\section{Subjects}

The study population consisted of 22 patients followed at the Cognitive Neurology Outpatient Clinic of the Hospital das Clínicas, Universidade Federal de Minas Gerais (HC-UFMG), in Belo Horizonte, Brazil. All patients fulfilled the diagnostic criteria of probabe $\mathrm{AD}$ from the National Institute of Neurological and Communicative Disorders and Stroke (NINCDS) and Alzheimer's disease and Related Disorders Association (ADRDA) ${ }^{16}$. Twenty-seven controls without any neurological signs and symptoms of dementia submitted to elective surgery and undergoing spinal anesthesia were also included. This study was approved by the Institutional ethics com- mittee for human subjects and all subjects, or their relatives when appropriate, provided informed written consent before admission to the study.

All participants were evaluated and had no infectious process, cancer, inflammatory or auto-immune diseases and were not taking any anti-inflammatory or immunosuppressive drugs. Information regarding medications use, Mini-Mental State Examination (MMSE) scores and the Functional Assessment Staging (FAST) were obtained for patients and controls.

\section{CSF sample collection}

The CSF was collected by lumbar puncture with subjects in lateral recumbency. The amount of material harvested was about $5 \mathrm{ml}$. All the assays were done by Enzyme-linked immunosorbent assay (ELISA).

Chemokine levels were measured by using the following kits: Human CCL2 DuoSet (DY279); Human CXCL10 DuoSet (DY266) e Human CXCL8 DuoSet (DY208) from R\&D Systems (Minneapolis, MN, USA) according to the manufacturer's protocol. The levels of beta-amyloid, tau and phospho-tau were determined in the CSF using the following kits: Innotest ${ }^{\circ}$ $\beta$-Amyloid(1-42); Innotest ${ }^{\circ}$ phospho-tau (181P) e Innotest ${ }^{\circ} h$ tau $\mathrm{Ag}$ from Innogenetics (Gent, Belgium).

\section{Statistical analysis}

The data were analyzed using ANOVA followed by Dunn's test for comparison between groups. Differences were considered significant for $\mathrm{p}<0.05$. Statistical analyses were performed using SPSS 16.0 software (SPSS Inc., Chicago, IL, USA). A p value $\leq 0.05$ was considered statistically significant.

\section{RESULTS}

An overview of the characteristics of the study population is described in in Table 1. The average age of the AD group was $74.7 \pm 10.2$ years, while the mean age of the control group was $64.4 \pm 11.1$ years. The AD group included 17 women and 5 men; control group was composed by 13 women and 15 men. The average duration of AD symptoms was 46.4 months and the median MMSE score was 16 for patients and 23 for controls.

Fig 1 shows the levels of $\mathrm{BA}$, tau and p-tau in the $\mathrm{CSF}$ in $\mathrm{AD}$ patients and controls. The levels of tau were higher in AD compared to controls (488.4 \pm 103 vs. $299.7 \pm 48.3$ ), while the levels of BA were decreased in AD group (304.8 \pm 46.6 vs. $335.2 \pm 44.1$ ). Only the p-tau levels were significantly increased in AD patients $(108.5 \pm 19.6)$ compared to controls $(67.2 \pm 4.1)$. The ratio tau/p-tau was significantly lower in $\mathrm{AD}$ group (3.3 \pm 0.5$)$ than controls (5.3 \pm 0.7$)$ (Fig 1).

Fig 2 shows the levels of chemokines in the CSF of 

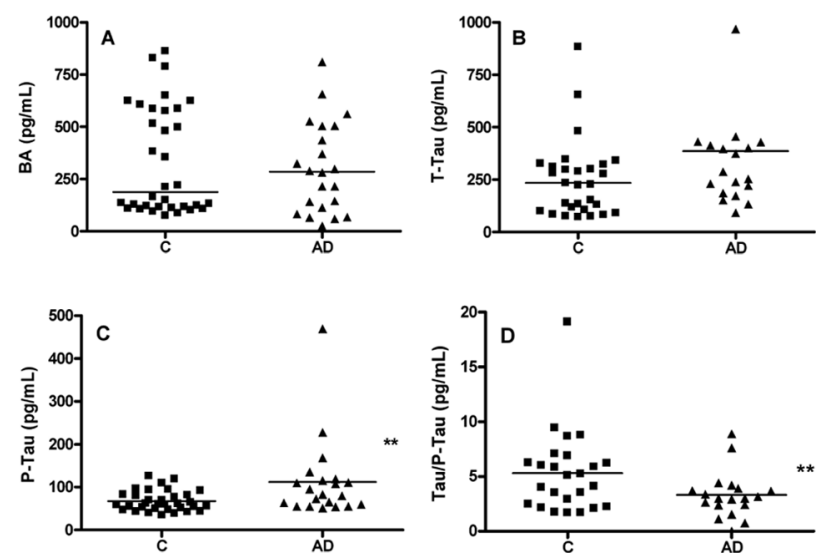

Fig 1. Levels of Beta-Amyloide (BA) [A], tau [B], phospho-tau (p-tau) $[C]$ and tau/p-tau ratio [D] in the CSF of patients with Alzheimer's disease (AD) and controls subjects (C). ${ }^{*}$ Statistically different from controls, $p<0.05$.

Table 1. Main clinical findings of patients.

\begin{tabular}{lcc}
\hline Clinical features & Control $(\mathbf{n}=27)$ & $\mathrm{AD}(\mathrm{n}=\mathbf{2 2})$ \\
\hline Gender & & \\
$\quad$ Male & $15(55.5 \%)$ & $5(22.7 \%)$ \\
$\quad$ Female & $12(44.5 \%)$ & $17(77.3 \%)$ \\
Age & $64.4 \pm 11.1$ & $74.7 \pm 10.2$ \\
Evolution (months) & $\mathrm{NA}$ & $46.4 \pm 20.8$ \\
Level of education (years) & $4.5 \pm 3.6$ & $4.6 \pm 3.4$ \\
MMSE & & \\
$\quad<20$ & $3(21.4 \%)$ & $14(63.6 \%)$ \\
$\quad 20$ & $11(78.6 \%)$ & $8(36.4 \%)$ \\
FAST & & \\
$\quad<6$ & $27(100 \%)$ & $14(63.6 \%)$ \\
$\geq 6$ & 0 & $8(36.4 \%)$ \\
IAche & 0 & $15(68.2)$ \\
$\quad$ Yes & $27(100 \%)$ & $7(31.8 \%)$ \\
No & 0
\end{tabular}

Data are expressed as mean \pm SD. *Disease duration was defined as the time in months between the first symptoms (by history) and the lumbar puncture. MMSE: mini-mental state scores; FAST: functional assessment staging; IAche: acetylcholinesterase inhibitors; NA: not applicable.
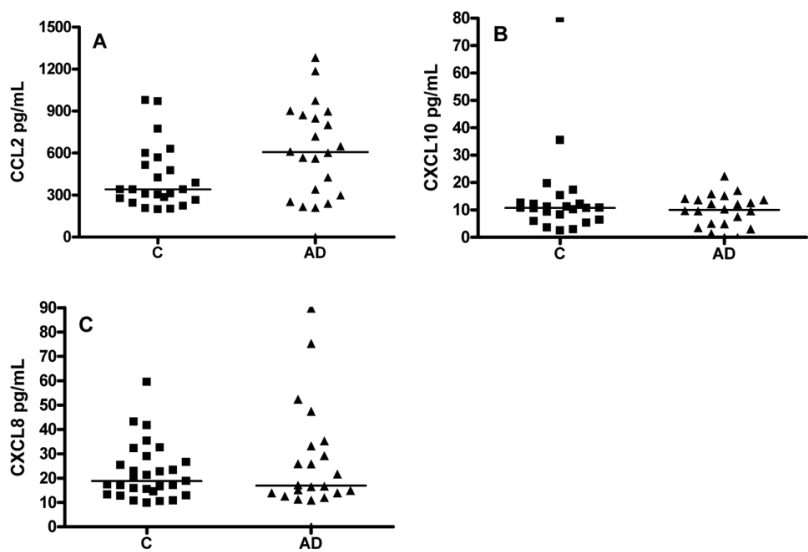

Fig 2. Levels of chemokines CCL2 [A], CXCL10 [B] and CXCL8 [C] in the CSF of patients with Alzheimer disease (AD) and controls subjects [C]. ${ }^{*}$ Stastistically different from controls, $p<0.05$.

AD patients and controls. It may be noted a significant increase in CCL2 (611.9 \pm 72.32 vs. $423.1 \pm 46.30$, respectively for AD and control subjects). The values of CXCL10 and CXCL8 did not differ between groups.

Table 2 summarizes the correlations between the levels of BA, tau and p-tau and chemokines in the CSF of AD patients. There was a significant positive correlation between levels of BA and all studied chemokines. In addition there was also a significant positive correlation between levels of $\mathrm{p}$-tau and CCL2.

No positive correlation was observed between the levels of BA, tau, p-tau, chemokines and clinical parameters.

Table 3 shows the correlations between MMSE and clinical variables. The MMSE showed a positive correlation with the progression of the disease, FAST and educational level.

\section{DISCUSSION}

The two primary cardinal lesions associated with $\mathrm{AD}$ are the neurofibrillary tangles and the neuritic plaques. The neurofibrillary tangles consist of abnormal accumu-
Table 2. Correlations between the levels of chemokines and beta-amyloid (BA), tau (T) and p-tau (phospho-tau) in CSF (cerebrospinal fluid).

\begin{tabular}{lcccccccc}
\hline & \multicolumn{3}{c}{ Control $(\mathrm{n}=27)$} & & \multicolumn{3}{c}{ AD $(\mathrm{n}=22)$} \\
\cline { 2 - 5 } \cline { 7 - 8 } & CCL2 & CXCL1 & CXCL10 & & CCL2 & CXCL1 & CXCL10 \\
\hline BA & $<0.001^{*}$ & 0.015 & NS & & 0.004 & 0.029 & 0.034 \\
& $(0.644)^{* *}$ & $(0.414)$ & & & $(0.592)$ & $(0.466)$ & $(0.453)$ \\
T-tau & NS & NS & NS & & NS & NS & NS \\
P-tau & NS & NS & NS & & 0.028 & NS & NS \\
& & & & & $(0.469)$ & & \\
T-tau/p-tau & 0.016 & NS & NS & & NS & NS & NS \\
& $(-0.438)$ & & & & & & \\
\hline
\end{tabular}

*P values. **Values in parenthesis represent the correlation coefficients. NS: not significant; AD: Alzheimer's disease.
Table 3. Correlation between MMSE and clinical parameters.

\begin{tabular}{|c|c|c|}
\hline MMSE versus & Control $(n=27)$ & $A D(n=22)$ \\
\hline Age & NS & NS \\
\hline Evolution & NA & $0.001^{*}(0.668)^{* *}$ \\
\hline IAche & NS & NS \\
\hline FAST & NS & $0.001(0.678)$ \\
\hline $\begin{array}{l}\text { Level of } \\
\text { education }\end{array}$ & $0.026(0.590)$ & $0.005(0.571)$ \\
\hline
\end{tabular}


lations of p-tau within the cytoplasm of certain neurons. The neuritic plaques consists of a central core of BA, surrounded by abnormally configured neuronal processes or neurites ${ }^{17}$.

Previous studies emphasized the utilization of CSF levels of tau, p-tau and BA or their ratios as biological markers to differentiate $\mathrm{AD}$ from healthy controls and also from other dementias ${ }^{6,7}$ Our results are consistent with previous findings showing increased levels of tau, $\mathrm{p}$-tau and lower levels of BA in CSF of AD patients ${ }^{18}$.

Immunoreactivity for some chemokines has been demonstrated in resident cells of the CNS, and the up regulation of some of them is associated with the pathological changes found in $\mathrm{AD}^{13}$. A growing body of evidence is implying inflammatory mechanisms and immune system alterations in the pathophisiology of $\mathrm{AD}^{2,8,19}$. In this setting, CXCL10, CCL2, and CXCL8 levels were evaluated in CSF of patients with AD and compared with age-matched controls. CSF levels of CCL2 and CXCL8 were higher in patients with AD, while CXCL10 levels were increased only in a subgroup of patients which MMSE score was higher than $15^{4}$.

In the present study, we also correlated the levels of chemokines with known markers of AD, such as BA, tau and $\mathrm{p}$-tau. We did not observe significant differences in CXCL8 and CXCL10 levels between controls and patients. Similar data were obtained in previous studies ${ }^{14,19}$.

We found that only the CSF levels of CCL2 were significant increased in subjects with AD compared to healthy controls. In accordance, previous studies showed immunohistochemical expression of CCL2 in mature, but not in immature, senile plaques and in reactive microglia of brain tissues from patients with $\mathrm{AD}^{20}$. This suggests that CCL2-related inflammatory events induced by reactive microglia contribute to the maturation of senile plaques ${ }^{19}$. CCL2 may also exert a role in the recruitment of phagocytic cells within the brain during the course of $\mathrm{AD}$, although activation of these cells may depend on the presence of other molecules, such as CXCL10 ${ }^{4}$.

We did find a positive correlation between levels of BA and levels of inflammatory mediators in CSF. The deposition of BA activates microglia to produce proinflammatory cytokines, which may also be responsible for the accumulation of chemokines in CSF${ }^{4}$. Teixeira et al. ${ }^{10}$ previously demonstrated that after exposure to BA, peripheral blood mononuclear cells from young healthy individuals had a significant increase of cytokine production. Furthermore, in vitro studies have demonstrated the ability of BA to stimulate the production of CXCL8, CCL2, CCL3, and CCL4 from human monocytes ${ }^{14}$.

Another interesting finding in our study is the positive correlation between the levels of CCL2 and p-tau in CSF. Tau is abnormally hyperphosphorylated in AD brain $^{21}$. The released hyperphosphorylated tau causes the disassembly of microtubules and the self-assembly of the hyperphosphorylated protein into neurofibrillary tangles. The disruption of the microtubules probably leads to neurodegeneration ${ }^{22}$. In a previous study, it was observed that high CSF p-tau levels correlated with cognitive decline and conversion from mild cognitive impairment to $\mathrm{AD}^{23}$. These data together may reinforce the involvement of CCL2 in disease progression and cognitive decline in $\mathrm{AD}$ patients. However, we did not observe positive correlation between the levels of BA, tau, p-tau and chemokines with clinical parameters.

We also found a positive correlation between the MMSE and FAST, which was expected because both instruments assess clinical parameters (cognition and function). The correlation of MMSE with the evolution of pathology is the same as expected, since the patient usually has a decline of their mental abilities over time ${ }^{17}$. The positive correlation between MMSE and level of education demonstrates a fact that has been studied with great interest. It appears that individuals with higher levels of education have less cognitive decline ${ }^{24-28}$.

In summary the study of chemokine profile in $\mathrm{AD}$ may lead to a better understanding of its pathological mechanisms. The evaluation of inflammatory mediators production in $\mathrm{AD}$ patients is also important in the search for a biomarkers for AD. The identification of AD biomarkers may allow an early, less invasive and more accurate diagnosis and be useful for monitoring disease progression and therapeutic efficacy.

\section{REFERENCES}

1. Olson L, Humpel C. Growth factors and cytokines/chemokines as surrogate biomarkers in cerebrospinal fluid and blood for diagnosing Alzheimer's disease and mild cognitive impairment. Exp Gerontol 2010;45:41-46.

2. Akiyama H, Barger S, Barnum S, et al. Inflammation and Alzheimer's disease. Neurobiol Aging 2000;21:383-421.

3. Bermejo P, Martin-Aragon S, Benedi J, et al. Differences of peripheral inflammatory markers between mild cognitive impairment and Alzheimer's disease. Immunol Lett 2008;117:198-202.

4. Galimberti D, Schoonenboom N, Scheltens P, et al. Intrathecal chemochine levels in Alzheimer disease and frontotemporal lobar degeneration. Neurology 2006;66:146-147.

5. Consensus report of the Working Group on: "Molecular and Biochemical Markers of Alzheimer's Disease". The Ronald and Nancy Reagan Research Institute of the Alzheimer's Association and the National Institute on Aging Working Group. Neurobiol Aging 1998;19:109-116.

6. Schuitemaker A, Dik MG, Veerhuis R, et al. Inflammatory markers in AD and $\mathrm{MCl}$ patients with different biomarker profiles. Neurobiol 2009;30: 1885-1889.

7. Sunderland T, Linker G, Mirza N, et al. Decreased beta-amyloid1-42 and increased Tau levels in cerebrospinal fluid of patients with Alzheimer disease. JAMA 2008;289:2094-2103.

8. Franceschi $C$, Valensin S, Bonafe $M$, et al. The network and the remodeling theories of aging, historical background and new perspectives. Exp Gerontol 2000;35:879-896.

9. Teixeira AL, Bauer ME, Nicolato R, Reis HJ, Palotás A. Cytokines and the brain: beyond immune response. In: Fedorovich SV (Ed). Signal Transduction in nervous Cells. Trivandrum, 2008:95-115. 
10. Teixeira AL, Reis HJ, Coelho FM, et al. All-or-nothing type biphasic cytokine production of human lymphocytes after exposure to Alzheimer's betaamyloid peptide. Biol Psychiatry 2008;64:891-895.

11. Tarkowski E, Andreasen N, Tarkowski A, Blennow K. Intrathecal inflammation precedes development of Alzheimer's disease. J Neurol Neurosurg Psychiatry 2003;74:1200-1205.

12. Baggiolini M, Dewald B, Moser B. Interleukin 8 and related chemotactic cytokines-CXC and CC chemokines. Adv Immunol 1994;55:97-179.

13. Lee KS, Chung JH, Choi TK, Suh SY, Oh BH, Hong CH. Peripheral cytokines and chemokines in Alzheimer's disease. Dement Geriatr Cogn Disord 2009;28:281-287.

14. Xia MQ, Hyman BT. Chemokines/chemokine receptors in the central nervous system and Alzheimer's disease. J Neurovirol 1999;5:32-41.

15. Galimberti D, Venturelli E, Fenoglio C, et al. IP-10 serum levels are not increased in mild cognitive impairment and Alzheimer's disease. Eur J Neurol 2007;14:3-4.

16. McKhann G, Drachman D, Folstein M, Katzman R, Price D, Stadlan EM. Clinical diagnosis of Alzheimer's disease: report of the NINCDS-ADRDA Work Group under the auspices of Department of Health and Human Services Task Force on Alzheimer's Disease. Neurology 1984;34:939-944.

17. Perl DP. Neuropathology of Alzheimer's disease. Mt Sinai J Med 2010;77: 32-42.

18. Ibach $B$, Binder $H$, Dragon $M$, et al. Cerebrospinal fluid tau and betaamyloid in Alzheimer patients, disease controls and an age-matched random sample. Neurobiol Aging 2006;27:1202-1211.

19. Bonotis K, Krikki E, Holeva V, Aggouridaki C, Costa V, Baloyannis S. Systemic immune aberrations in Alzheimer's disease patients. J Neuroimmunol 2008; 193:183-187.

20. Ishizuka K, Kimura T, Igata-yi R, Katsuragi S, Takamatsu J, Miyakawa T. Identification of monocyte chemoattractant protein-1 in senile plaques and reactive microglia of Alzheimer's disease. Psychiatry Clin Neurosci 1997;51:135-138.

21. Iqbal K, Liu F, Gong CX, Alonso Adel C, Grundke-lqbal I. Mechanisms of tau-induced neurodegeneration. Acta Neuropathol 2009;118:53-69.

22. Alonso AC, Iqbal IG, Barra H S, Iqbal K. Abnormal phosphorylation of tau and the mechanism of Alzheimer neurofibrillary degeneration: sequestration of microtubule-associated proteins 1 and 2 and the disassembly of microtubules by the abnormal Tau. Proc Natl Acad Sci U S A 1997;94: 298-303.

23. Buerger K, Teipel SJ, Zinkowski R, et al. CSF tau protein phosphorylated at threonine 231 correlates with cognitive decline in $\mathrm{MCl}$ subjects. Neurology 2002;59:627-629.

24. Aevarsson O, Skoog IA. Longitudinal population study of the mini-mental state examination in the very old: relation to dementia and education. Dement Geriatr Cogn Disord 2000;11:166-175.

25. Brayne $C$, Ince $P G$, Keage $H A$, et al. Education, the brain and dementia: neuroprotection or compensation? Brain 2010;133:2210-2216.

26. Katzman R. Education and the prevalence of dementia and Alzheimer's disease. Neurology 1993;43:13-20.

27. Stern $Y$. What is cognitive reserve? Theory and research application of the reserve concept. J Int Neuropsychol Soc 2002;8:448-460.

28. Stern Y. Cognitive reserve and Alzheimer disease. Alzheimer Dis Assoc Disord 2006;20:69-74. 